\title{
Characteristics of Chronic Hepatitis B Virus Patients Related Liver Cirrhosis in a Tertiary Care Referral Hospital, Duhok, Iraqi Kurdistan
}

\author{
Muayad A Merza* \\ Azadi Teaching Hospital, Department of Internal Medicine, College of Medicine, University of Duhok, Duhok, Iraqi \\ Kurdistan
}

Received: April 3, 2017; Accepted: May 30 , 2017; Published: June 8, 2017

*Corresponding author:Muayad A Merza, Azadi Teaching Hospital, Department of Internal Medicine, College of Medicine, University of Duhok, Azadi Hospital Street, Duhuk, Kurdistan, Iraq, Tel: +964-750-4506172; Fax: +964-62-761-8824; E-mail: muayad.merza@uod.ac

\begin{abstract}
Background: Chronic hepatitis B virus (HBV) related to liver cirrhosis is an advanced liver fibrosis that is usually progressive, irreversible, and the only option for the treatment is liver transplantation in selected patients

Objectives: to examine the demographic, clinical, and laboratory characteristics of patients with the chronic HBV related to cirrhosis.

Methods: The viral hepatitis clinic in Azadi Teaching Hospital is dealing with all viral hepatitis cases. All patients with liver cirrhosis caused by chronic HBV infection were studied. Demographic, clinical and laboratory information of the patients were retrieved by interviewing and from the case notes of September in 2014 until December in 2016. Child-Turcotte-Pugh (CTP) classification was used to evaluate the prognosis of liver cirrhosis. The results obtained were analyzed by entering the data into a Microsoft Excel spreadsheet. A value of less than 0.05 was considered as statistically significant.
\end{abstract}

Results: The total number of patients included in the study was 45. The mean age of the patients was $53.73 \pm 14.23$ years. Thirtysix cases were males and 9 were females. The evaluation of the 45 patients revealed a higher frequency of CTP-A in both male and female sexes. Cirrhotic patients classified as CTP-A $(n=30)$ and $B(n=12)$ were compared by demographic, clinical and laboratory characteristics. Patients classified as CTP-B were significantly associated with older ages. The levels of hemoglobin, WBC, and platelet were lower, but prothrombin time was higher. There were also significant associations with higher total serum bilirubin (TSB), AST, and ALP levels; whereas, significant associations with the lower levels of albumin, and triglyceride was noted in Child's B. The AST/ALT ratio of 1 or more was significantly associated with CTP-B.

Conclusions: The cirrhotic patients with CTP-B were older, lower weighted, lower hemoglobin level, lower WBC count, lower platelet counts, increased prothrombin time, increased TSB level, decreased serum albumin level, decreased triglyceride level, increased AST level, AST/ALT ratio ( $\geq 1)$, and increased ALP level.

Key words: Cirrhosis; Chronic Hepatitis B; Characteristic

\section{Introduction}

Despite the availability of vaccination against hepatitis B virus (HBV) and antiviral treatment, more than one million deaths occurred in the world due to liver cirrhosis [1]. Liver cirrhosis is an advanced liver fibrosis that is usually progressive, irreversible, and the only option for the treatment is liver transplantation in selected patients. The leading risk factors of liver cirrhosis include alcohol consumption, non-alcoholic steatohepatitis (NASH), HBV and hepatitis C virus infections [2]. In Asia, HBV comprises around $44 \%$ cases of liver cirrhosis [1]. There is no precise data about the prevalence of HBV related to cirrhosis in Iraq. On the other hand, the prevalence of HBV infection is estimated to be $1.6 \%$ in Iraq as a country [3] and $1.79 \%$ in Duhok as a province [4]. Generally, the development of cirrhosis is insidious and asymptomatic in more than $80 \%$ cases; whereas, the minority shows symptoms at the diagnosis [5]. The progression of HBV infection into liver cirrhosis is associated with some risk factors. For e.g. increasing age, male gender, alcohol intake, and co-infection with hepatitis $\mathrm{C}$ virus (HCV) [6]. There is diversity in clinical presentations of liver cirrhosis related to hepatitis B infection, ranging from asymptomatic to hepatocellular or portal hypertension manifestations [7]. In cirrhotic patients, the severity of liver disease is assessed by the Child-Turcotte-Pugh (CTP) score and the model for end stage liver disease (MELD) [8]. Child-Pugh is a commonly used as a scoring system, which is based on calculations of variables including total serum bilirubin (TSB), serum albumin, international normalized ratio (INR) or prothrombin time (PT), hepatic encephalopathy, and ascites [9]. To the best of our knowledge, this is the first study about characteristics of HBV infection related to cirrhosis in Iraq. The present study was aimed to examine the demographic, clinical, and laboratory characteristics of patients with the chronic HBV related to cirrhosis.

\section{Patients and Methods}

\section{Setting}

The viral hepatitis clinic in Azadi Teaching Hospital is dealing with all viral hepatitis cases. The center is designed to manage viral hepatitis cases. The patients were visiting the center on regular intervals based on their clinical conditions. All of the patients' information was collected in standardized case 
notes.

\section{Study design and patients}

The study included all patients of liver cirrhosis caused by chronic HBV infection. Demographic, clinical and laboratory information of the patients were retrieved by interviewing and from the case notes of September in 2014 until December in 2016. Child-Pugh's classification was used to evaluate the prognosis of liver cirrhosis (10). The classification has 3 grades. The study was approved by the ethical research committee, College of Medicine, University of Duhok. An informed written consent was obtained from all participants in this study. The inclusion criteria included hepatitis B surface antigen (HBsAg) positive patients, Anti-hepatitis B core antigen antibodies-IgG positive with liver cirrhosis. The diagnosis of liver cirrhosis was based on either laboratory findings, clinical findings of portal hypertension, imaging findings including ultrasound, endoscopic features of varices of esophagus or stomach and/or histological findings. All cases of liver cirrhosis due to causes other than HBV were excluded from the study.

\section{Statistical analysis}

The results obtained were analyzed by entering the data into a Microsoft Excel spreadsheet. A value of less than 0.05 was considered as statistically significant.

\section{Results}

The total number of patients included in the study was 45. The mean age of the patients was $53.73 \pm 14.23$ years. Thirtysix cases were males and 9 were females with a male: female ratio of $4: 1$. Baseline characteristics of the study population are demonstrated in table 1 . Severity of liver disease on presentation was evaluated using Child-Pugh's score (Figure). The evaluation of the 45 patients revealed a higher frequency of Child-Pugh's A classification in both male and female sexes, which comprised the same percentage $(66.7 \%)$ for both genders (Table 2 ).

Cirrhotic patients classified as Child's grade A $(n=30)$ and $B(n=12)$ were compared by age, gender, ethnicity, body mass

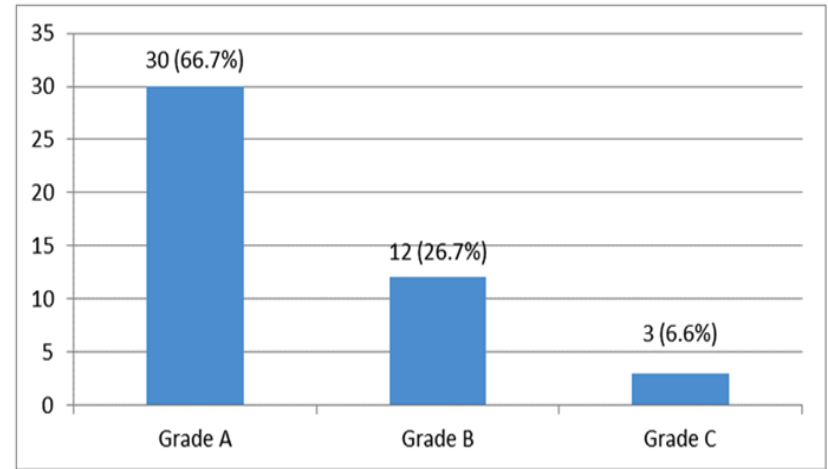

Figure: Child-Pugh's classification in studied population
Table 1: Clinico-demogarphic and laboratory profile of chronic hepatitis $B$ virus patients related liver cirrhosis

\begin{tabular}{|c|c|c|}
\hline \multicolumn{2}{|c|}{ Variable } & MEAN \pm SD $($ no $=45)$ \\
\hline \multicolumn{2}{|c|}{ Age } & $53.73 \pm 14.23$ \\
\hline \multirow{2}{*}{ Sex } & Male & $36(80 \%)$ \\
\hline & Female & $9(20 \%)$ \\
\hline \multirow{2}{*}{ Ethinicity } & Iraqi & $39(86.7 \%)$ \\
\hline & Syrian & $6(12.3 \%)$ \\
\hline \multicolumn{2}{|c|}{ Weight (kg) } & $78.93 \pm 14.28$ \\
\hline \multicolumn{2}{|c|}{ Height (cm) } & $170.74 \pm 14.28$ \\
\hline \multicolumn{2}{|c|}{$\mathrm{BMI} \mathrm{kg} / \mathrm{m} 2$} & $27.13 \pm 4.23$ \\
\hline \multicolumn{2}{|c|}{ Hemoglobin gm/dl } & $14.63 \pm 1.78$ \\
\hline \multicolumn{2}{|c|}{ WBC (mm3) } & $5566.0 \pm 1613.74$ \\
\hline \multicolumn{2}{|c|}{ Platelet count (mm3) } & $154355.56 \pm 73787.43$ \\
\hline \multicolumn{2}{|c|}{ Prothrombin time (second) } & $14.91 \pm 2.20$ \\
\hline \multicolumn{2}{|c|}{ TSB mg/dl } & $1.6 \pm 0.72$ \\
\hline \multicolumn{2}{|c|}{ Albumin gm/dl } & $3.22 \pm 0.32$ \\
\hline \multicolumn{2}{|c|}{ Protein gm/dl } & $7.21 \pm 0.83$ \\
\hline \multicolumn{2}{|c|}{ FBS mg/dl } & $96.11 \pm 18.45$ \\
\hline \multicolumn{2}{|c|}{ Cholesterol mg/dl } & $171.16 \pm 43.43$ \\
\hline \multicolumn{2}{|c|}{ Triglyceride mg/dl } & $131.62 \pm 67.91$ \\
\hline \multicolumn{2}{|c|}{ ALT U/l } & $48.18 \pm 27.47$ \\
\hline \multicolumn{2}{|c|}{ AST U/l } & $68.58 \pm 36.18$ \\
\hline \multicolumn{2}{|c|}{ ALP U/l } & $283.1 \pm 53.94$ \\
\hline \multicolumn{2}{|c|}{ AFP ng/ml } & $3.78 \pm 4.30$ \\
\hline \multicolumn{2}{|c|}{ HBV-DNA viral load IU/ml } & $21232.32 \pm 32585.13$ \\
\hline
\end{tabular}

Note: $\mathrm{BMI}=$ body mass index, $\mathrm{WBC}=$ white blood cell, $\mathrm{TSB}=$ total serum bilirubin, FBS = fasting blood sugar, ALT = alanine amino transferase, AST $=$ aspartate aminotransferase, ALP $=$ alkaline phosphatase, AFP = alpha-fetoprotein, HBV = hepatitis B virus.

index (BMI), hematological indices, biochemistry parameters, viral markers, and viral load (Table 3). Patients classified as Child's $\mathrm{B}$ grade were significantly associated with older ages. The levels of hemoglobin, WBC, and platelet were lower, but prothrombin time was higher. There were also significant associations with higher total serum bilirubin (TSB), AST, and ALP levels; whereas, significant associations with the lower levels of albumin, and triglyceride was noted in Child's B. The AST/ALT ratio of 1 or more was significantly associated with Child's grade B (Table 3). Other parameters did not show significant associations. During the study period, the prognosis of the patients in CTP-A and CTP-B were good; whereas, the prognosis of the patients in CTP-C were poor. As a proof, two patients died because of the development of hepatocellular carcinoma (HCC). 
Table 2: Child-Pugh Classification of chronic hepatitis B virus related cirrhosis

\begin{tabular}{|c|c|c|c|}
\hline Child-Pugh score & Male (no=36) no (\%) & Female (no =9) no (\%) & Total (no=45) no (\%) \\
\hline Grade A & $24(66.7)$ & $6(66.7)$ & $30(66.7)$ \\
\hline Grade B & $9(25.0)$ & $3(33.3)$ & $12(26.7)$ \\
\hline Grade C & $3(8.3)$ & $0(0.0)$ & $3(6.6)$ \\
\hline
\end{tabular}

Table 3: Comparison of the characteristics between the 42 patients with Child-Pugh classes A and B

\begin{tabular}{|c|c|c|c|c|c|}
\hline \multicolumn{2}{|c|}{ Parameter } & $\begin{array}{c}\text { Child-Pugh A } \\
\text { (no=30) } \\
\text { no (\%) }\end{array}$ & $\begin{array}{c}\text { Child-Pugh B } \\
\text { (no=12) } \\
\text { no (\%) }\end{array}$ & OR $(95 \% \mathrm{CI})$ & $P$ value \\
\hline \multicolumn{2}{|c|}{ Age (year) } & $49.67 \pm 14.44$ & $62.75 \pm 10.25$ & $-13.08(-22.3434$ to -3.8166$)$ & 0.0068 \\
\hline \multirow{2}{*}{ Sex } & Male & 26 & 9 & \multirow{2}{*}{$1.444(0.225$ to 8.799$)$} & 0.687 \\
\hline & Female & 6 & 3 & & \\
\hline \multirow{2}{*}{ Ethinicity } & Iraqi & 27 & 10 & \multirow{2}{*}{$0.556(0.060$ to 5.687$)$} & 0.613 \\
\hline & Syrian & 3 & 2 & & \\
\hline \multicolumn{2}{|c|}{ Weight (kg) } & $82.33 \pm 14.40$ & $71.17 \pm 10.50$ & 11.16 (1.8814 to 20.4386$)$ & 0.0196 \\
\hline \multicolumn{2}{|c|}{ Height $(\mathrm{cm})$} & $172.10 \pm 7.88$ & $167.45 \pm 5.82$ & $4.6500(-0.4385$ to 9.7385$)$ & 0.0722 \\
\hline \multicolumn{2}{|c|}{$\mathrm{BMI} \mathrm{kg} / \mathrm{m} 2$} & $27.8 \pm 4.32$ & $25.48 \pm 3.52$ & $2.3200(-0.5211$ to 5.1611$)$ & 0.1067 \\
\hline \multicolumn{2}{|c|}{ Hemoglobin gm/dl } & $15.01 \pm 1.80$ & $13.64 \pm 1.49$ & $1.3700(0.1824$ to 2.5576$)$ & 0.0249 \\
\hline \multicolumn{2}{|c|}{ WBC (mm3) } & $6006.67 \pm 1124.85$ & $4839.17 \pm 2173.18$ & $\begin{array}{c}1167.50(139.8427 \text { to } \\
2195.1573)\end{array}$ & 0.027 \\
\hline \multicolumn{2}{|c|}{ Platelet count (mm3) } & $187200.0 \pm 66401.0$ & $89500.0 \pm 33006.31$ & $\begin{array}{c}97700.0(56881.9096 \text { to } \\
138518.0904)\end{array}$ & 0.0001 \\
\hline \multicolumn{2}{|c|}{ Prothrombin time (second) } & $13.8 \pm 1.01$ & $16.42 \pm 1.89$ & $-2.6200(-3.5259$ to -1.7141$)$ & 0.0001 \\
\hline \multicolumn{2}{|c|}{ TSB mg/dl } & $1.15 \pm 0.11$ & $2.24 \pm 0.19$ & -1.09 (1.1844 to -0.9956$)$ & 0.0001 \\
\hline \multicolumn{2}{|c|}{ Albumin gm/dl } & $3.35 \pm 0.21$ & $3.06 \pm 0.22$ & $0.29(0.1431$ to 0.4369$)$ & 0.0003 \\
\hline \multicolumn{2}{|c|}{ Protein gm/dl } & $7.29 \pm 0.93$ & $6.92 \pm 0.50$ & $0.3700(-0.2058$ to 0.9458$)$ & 0.2015 \\
\hline \multicolumn{2}{|c|}{ FBS mg/dl } & $97.93 \pm 20.11$ & $92.75 \pm 14.83$ & $5.1800(-7.8026$ to 18.1626$)$ & 0.4248 \\
\hline \multicolumn{2}{|c|}{ Cholesterol mg/dl } & $178.97 \pm 44.68$ & $163.58 \pm 34.24$ & $15.39(-13.6508$ to 44.4308$)$ & 0.2906 \\
\hline \multicolumn{2}{|c|}{ Triglyceride mg/dl } & $145.13 \pm 73.28$ & $98.67 \pm 44.09$ & $46.46(0.5244$ to 92.3956$)$ & 0.0476 \\
\hline \multicolumn{2}{|c|}{ ALT U/l } & $49.73 \pm 28.68$ & $48.17 \pm 26.45$ & $1.56(-17.8275$ to 20.9475$)$ & 0.8716 \\
\hline \multicolumn{2}{|c|}{ AST U/l } & $60.83 \pm 29.90$ & $86.42 \pm 46.52$ & $-25.59(-49.9312$ to -1.2488$)$ & 0.0398 \\
\hline \multicolumn{2}{|c|}{ AST/ALT ratio $(\geq 1)$} & $1 / 30$ & $6 / 12$ & $29.0(2.511$ to 776.224$)$ & 0.001 \\
\hline \multicolumn{2}{|c|}{ ALP U/l } & $191.65 \pm 116.51$ & $291.0 \pm 59.30$ & $-99.35(-171.1196$ to -27.5804$)$ & 0.0079 \\
\hline \multicolumn{2}{|c|}{ AFP ng/ml } & $3.17 \pm 3.70$ & $6.05 \pm 31.94$ & $-2.88(-14.6454$ to 8.8854$)$ & 0.6235 \\
\hline \multicolumn{2}{|c|}{ HBsAg positive } & $30 / 30$ & $12 / 12$ & - & - \\
\hline \multicolumn{2}{|c|}{ Anti-HBc total positive } & $30 / 30$ & $12 / 12$ & - & - \\
\hline \multicolumn{2}{|c|}{ HBeAg positive } & $5 / 30$ & $0 / 12$ & Inf (0339 to Inf) & 0.298 \\
\hline \multicolumn{2}{|c|}{ Anti-Hbe positive } & $20 / 30$ & $10 / 12$ & $0.40(0.049$ to 2.60$)$ & 0.453 \\
\hline \multicolumn{2}{|c|}{ HBV-DNA viral load IU/ml } & $22739.70 \pm 32970.62$ & $12698.67 \pm 27141.20$ & $\begin{array}{c}10041.03(-11687.2887 \text { to } \\
31769.3487)\end{array}$ & 0.3559 \\
\hline
\end{tabular}




\section{Discussion}

Hepatitis B related to cirrhosis is directly linked with morbidity and mortality in chronic HBV infection. No age exempt exists for liver cirrhosis in chronic HBV. In this study, the mean age of the patients was $53.73 \pm 14.23$ years, which is consistent with other literatures that document frequent occurrence of liver cirrhosis in old patients [11]. In the current study, there were predominance of both male sex (80\%) and Iraqi nationality (86.7\%). The high rate of male ratio is in parallel with other studies, which documents a higher rate exposure of men to HBV infection [12]. In our previous work in 2016, we studied the prevalence of hepatitis $\mathrm{B}$ and $\mathrm{C}$ in 214 tuberculosis patients. We found that $97.2 \%$ were Iraqi and $2.8 \%$ were mainly Syrian nationality [13]. Perhaps, the high rate of the Syrian patients $(12.3 \%)$ in the present study has resulted from the fled of the people to Iraqi Kurdistan following internal conflicts in Syria. In addition, Syria is an intermediate HBV incidence country. In this study, we classified cirrhotic patients into three groups according to Child-Pugh's scoring system [10]. Out of 45 patients, 30 (66.7\%), 12 (26.7\%), and $3(6.6 \%)$ patients were graded as Child's A, B, and C, respectively. The figure was in line with other studies [14]. This can be justified for hepatitis B patients as they undergo regular medical follow ups, which makes earlier detection of liver cirrhosis. In contrast, the prevalence of Child's B is higher in alcoholic cirrhosis than HBV etiology, which may be explained by alcoholic patients as they seek medical advices after the appearance of clinical symptoms.

In order to show association between clinico-demographic and laboratory profile with the severity of hepatic cirrhosis, CTP-A was compared with CTP-B. In this study, there was a significant association between the severity of hepatic cirrhosis and the older age $(p=0.0068)$, lower weight $(p=0.0196)$, low hemoglobin $(\mathrm{p}=0.0249)$, low WBC $(\mathrm{p}=0.027)$, low platelet $(\mathrm{p}=0.0001)$, high prothrombin time $(\mathrm{p}=0.0001)$, increased TSB $(\mathrm{p}=0.0001)$, decreased serum albumin $(\mathrm{p}=0.0003)$, decreased triglyceride $(p=0.0476)$, increased AST $(p=0.0398)$, AST/ALT ratio $(\geq 1)(p \geq 0.001)$, and increased ALP $(p=0079)$. However, a significant statistical association was not found between the severity of hepatic cirrhosis and $\operatorname{sex}(p=0.687)$, ethnicity $(p=0.613)$, FBS $(p=0.4248)$, serum protein $(p=0.2015)$, ALT $(p=0.8716)$ AFP ( $p=0.6235)$, HBeAg positivity ( $p=0.298)$, anti-HBe positive $(\mathrm{p}=0.453)$, and viral load $(\mathrm{p}=0.3559)$. Similar to the findings in the present study, van der Plas et al. [15] reported more frequent liver cirrhosis among older age and men gender. Although, there were more severe hepatic cirrhotic men patients in this study, the association was not significant $(\mathrm{p}=0.687)$. In the current study, patients with Child-Pugh score B were significantly associated with lower body weight $(\mathrm{p}=0.0196)$, which was consistent with the finding of Li et al. [16]. Generally, in this study, patients with higher BMI were linked with higher albumin level and better liver enzymes, which results mild hepatic cirrhosis (Child's A). Furthermore, it is well known that the muscle mass is decreasing as liver cirrhosis progresses. In over all, the patients were overweight in this study, which was consistent with the finding of BMI of other literatures [17]. In contrast, Li et al. reported that the majority of the patients in their study were either underweight or normal weight (57\%) [16]. The discrepancy in the result between this study and other studies can be explained by the higher rate of CTP-A in my study, while higher rate of CTP-B or C in other studies. The hemoglobin, platelet and WBC levels were significantly lower in Child's B group in comparison to Child's A in the study population. It is well known that the chronic HBV infection is a recognized factor of pancytopenia in cirrhotic patients [18]. As the severity of hepatic impairment increases, portal hypertension and splenomegaly is increased as well, while thrombopoietin production is decreased, this subsequently results in more severe hematological abnormalities. Our results showed that prothrombin time, and TSB were significantly higher among Child's B, whereas the value of the albumin was significantly lower. These findings were in agreement with other literatures [19]. Liver is the only organ for synthesis of albumin and thus liver cirrhosis impairs albumin synthesis. Hence, the rate of albumin synthesis is directly lined with Child-Pugh's score [20]. In this study, serum triglyceride level was significantly lower among Child's B than Child's A population $(\mathrm{p}=0.0476)$. However, serum cholesterol level did not show association between the two groups. This figure has e.g. been reported by Kwarteng et al. who found serum triglyceride concentration with the lowest level among the chronic symptomatic HBV population, while serum cholesterol levels were unaffected markedly [21]. It is well described that following HBV infection, the secretion of triglycerides by the liver decreases, which subsequently results in steatotic liver condition [22]. In the current study, we found a significantly higher value of AST in Child's B group than child's A group ( $\mathrm{p}=0.398$ ); and similarly, the AST/ALT ratio was more than 1 or higher in cirrhotic Child's B than Child's $A(p=0.001)$. These figures were in line with other studies [23]. It is well known that as chronic liver disease is getting worse, the AST/ALT ratio is in increasing. Although the exact reason is not clear for this finding; however, it has been suggested that clearance of AST by sinusoidal cells of the liver is decreasing as liver cirrhosis progresses [24]. Furthermore, we found that ALP level was significantly higher among Child's B than Child's A group. In contrast to my finding, Das et al. [25] reported that ALP level remains normal in cirrhotic patients. Recent reports documented that the hypoalbuminemia and elevated ALP level in chronic liver disease is associated with poor overall survival. Therefore, the high risk albumin to alkaline phosphatase ratio (AAPR) is associated with poor prognostic feature, which includes CTP-B and C score [26]. In this study, 2 patients died from HCC in CTP-C. It is well known that HCC is the first cause of death among cirrhotic patients [27].

Although the duration of my study was 27 months, the sample size was small. Furthermore, I did not include some important parameters such as HDV co-infection, and GGT level, because these investigations were not available regularly throughout the study periods.

In conclusion, the current study investigated the associations between variables among CTP categories of chronic HBV patients related to liver cirrhosis in Duhok province. The cirrhotic patients with CTP-B were older, lower weighted, lower hemoglobin level, lower WBC count, lower platelet counts, increased pro- 
thrombin time, increased TSB level, decreased serum albumin level, decreased triglyceride level, increased AST level, AST/ALT ratio ( $\geq 1$ ), and increased ALP level. Although no significant association was found between HBV related to cirrhosis and other variables such as viral load, a larger cohort study with extended parameters are warranted to ascertain the association with severity of the hepatic cirrhosis.

\section{Acknowledgements}

We would like to thank all staff of the viral hepatitis clinic in Azadi Teaching Hospital, Duhok province for their kind help and support in performing this study.

\section{Funding/Support}

The viral hepatitis clinic in coordination with central laboratory in Duhok, performed patient management and laboratory investigations.

\section{References}

1. Mokdad AA, Lopez AD, Shahraz S, Lozano R, Mokdad AH, Stanaway J, et al. Liver cirrhosis mortality in 187 countries between 1980 and 2010: a systematic analysis. BMC Med. 2014;12:145. doi: 10.1186/s12916014-0145-y

2. Bosetti C, Levi F, Lucchini F, Zatonski WA, Negri E, La Vecchia C. Worldwide mortality from cirrhosis: an update to 2002. J Hepatol. 2007;46(5):827-839. doi:10.1016/j.jhep.2007.01.025

3. World Health Organization, Global policy report on the prevention and control of viral hepatitis. HIV/AIDS. 2013;220.

4. Merza MA, Hassan WM, Muhammad AS. Frequency of HBV and HCV among patients undergoing elective surgery in a tertiary care referral hospital in Duhok, Iraqi Kurdistan. JMSCR. 2014;2(7):1810-1815.

5. Realdi G, Fattovich G, Hadziyannis S, Schalm SW, Almasio P, SanchezTapias J, et al. Survival and prognostic factors in 366 patients with compensated cirrhosis type B: a multicenter study. The Investigators of the European Concerted Action on Viral Hepatitis (EUROHEP). J Hepatol. 1994;21(4):656-666. doi: dx.doi.org/10.1016/S01688278(94)80115-0

6. Yim HJ, Lok ASF. Natural history of chronic hepatitis B virus infection: What we knew in 1981 and what we know in 2005. Hepatology. 2006;43(2 suppl 1):S173-S181. doi:10.1002/hep.20956

7. Lertpipopmetha K, Auewarakul CU. High incidence of hepatitis B infection-associated cirrhosis and hepatocellular carcinoma in the Southeast Asian patients with portal vein thrombosis. BMC Gastroenterol. 2011;11:66. doi: 10.1186/1471-230X-11-66

8. Peng CY, Chien RN, Liaw YF. Hepatitis B virus-related decompensated liver cirrhosis: benefits of antiviral therapy. J Hepatol. 2012;57(2):442450. doi: 10.1016/j.jhep.2012.02.033

9. Pugh RN, Murray-Lyon IM, Dawson JL, Pietroni MC, Williams R. Transection of the oesophagus for bleeding oesophageal varices. Br J Surg. 1973; 60(8):646-649. doi: 10.1002/bjs.1800600817

10. Desmet VJ, Gerber M, Hoofnagle JH, Manns M, Scheuer PJ. Classification of chronic hepatitis: diagnosis, grading and staging. Hepatology. 1994;19(6):1513-1520. doi: 10.1002/hep.1840190629

11. Maskey R, Karki P, Ahmed SV, Manandhar DN. Clinical profile of patients with cirrhosis of liver in a tertiary care hospital, Dharan, Nepal. Nepal Med Coll J. 2011;13(2):115-118.
12. Stroffolini T, Esvan R, Biliotti E, Sagnelli E, Gaeta GB, Almasio PL. Gender differences in chronic HBsAg carriers in Italy: evidence for the independent role of male sex in severity of liver disease. J Med Virol. 2015;87(11):1899-1903. doi:10.1002/jmv.24243

13. Merza MA, Haji SM, Alsharafani AM, Muhammed SU. Low prevalence of hepatitis $\mathrm{B}$ and $\mathrm{C}$ among tuberculosis patients in Duhok Province, Kurdistan: Are HBsAg and anti-HCV prerequisite screening parameters in tuberculosis control program? Int J Mycobacteriol. 2016;5(3):313317. doi: 10.1016/j.ijmyco.2016.06.019

14. Stroffolini T, Sagnelli E, Gaeta GB, Sagnelli C, Andriulli A, Brancaccio G, et al. Characteristics of liver cirrhosis in Italy: Evidence for a decreasing role of HCV aetiology. Eur J Intern Med. 2017;38:68-72. doi: 10.1016/j.ejim.2016.10.012

15. van der Plas SM, Hansen BE, de Boer JB, Stijnen T, Passchier J, de Man $\mathrm{RA}$, et al. Generic and disease-specific health related quality of life in non-cirrhotic, cirrhotic and transplanted liver patients: a cross sectional study. BMC Gastroenterol. 2003,17;3:33. doi:10.1186/1471230X-3-33

16. Li Q Xing H, Liu D, Li H. Negative impact of low body mass index on liver cirrhosis patients with hepatocellular carcinoma. World J Surg Oncol. 2015;13:294. doi: 10.1186/s12957-015-0713-4

17. Paul S, O’Regan K, St John A, Stuart K, Crawford D. Interaction of serum ferritin and body mass index in patients with chronic hepatitis B: improved prediction of cirrhosis. Hepatology. 2013;57(5):2094-2095. doi: 10.1002/hep.26076

18. Qamar AA, Grace ND. Abnormal hematological indices in cirrhosis. Can J Gastroenterol. 2009;23(6):441-445. doi: dx.doi. org/10.1155/2009/591317

19. Temel T, Cansu DU, Temel HE, Ozakyol AH. Serum thrombopoietin levels and its relationship with thrombocytopenia in patients with cirrhosis. Hepat Mon. 2014;14(5):e18556. doi: 10.5812/hepatmon.18556

20. Anderson GF, Barnhart MI. Intracellular localization of prothrombin. Proc Soc Exp Biol Med. 1964;116:1-4.

21. Kwarteng J, Owusu L, Afihene M, E Mica, O Opare-Sem, FKN Arthur. Lowered serum triglyceride levels among chronic hepatitis B-infected patients in Ghana. J Sci Technol. 2012;32(3):1-10. doi: 10.4314/just. v32i3.1

22. Altiparmak E, Koklu S, Yalinkilic M, Yuksel O, Cicek B. Viral and host causes of fatty liver in chronic hepatitis B. World J Gastroenterol. 2005;11(20): 3056-3059. doi: 10.3748/wjg.v11.i20.3056

23. Williams AL, Hoofnagle JH. Ratio of serum aspartate to alanine aminotransferase in chronic hepatitis. Relationship to cirrhosis. Gastroenterology. 1988;95(3):734-739. doi: 10.1016/S0016-5085(88)800222

24. Giannini E, Risso D, Botta F, Chiarbonello B, Fasoli A, Malfatti F, et al. Validity and clinical utility of the aspartate aminotransferase-alanine aminotransferase ratio in assessing disease severity and prognosis in patients with hepatitis $C$ virus-related chronic liver disease. Arch Intern Med. 2003;163(2):218-224. doi: 10.1001/archinte.163.2.218

25. Das N, Bhattacharyya A, Paria B, Sarkar S. Study on assessment of renal function in chronic liver disease. J Clin Diagn Res. 2015;9(3):0C0912. doi:10.7860/JCDR/2015/11423.5658

26. Chan AW, Chan SL, Mo FK, Wong GL, Wong VW, Cheung YS, et al. Albumin-to-alkaline phosphatase ratio: a novel prognostic index for hepatocellular carcinoma. Dis Markers. 2015;2015:564057. doi: $10.1155 / 2015 / 564057$

27. Bruix J, Llovet JM. Hepatitis B virus and hepatocellular carcinoma. J Hepatol. 2003;39 Suppl 1:S59-63. 